\title{
Detecting Activated Thrombin Activatable Fibrinolysis Inhibitor (TAFIa) and Inactivated TAFIa (TAFIai) in Nomal and Hemophilia A Plasmas
}

\author{
John P. Hulme and Seong Soo A. An \\ Kutmgion Lnversity: Department of Bionano Technologv, Sungnam, Gveonghi f01-761, Korea \\ - E-mails: johnhlmeakwhgwon ackn; seongaaniákwngwon ack \\ Received September 17, 2008, Accepted November 6, 2008
}

\begin{abstract}
Thrombin activatable fibrinoly sis inhibitor (TAFI) also known as plasma procarboxypeptidase B or U is a $60 \mathrm{kD}$ glycoprotein, which is the major modulator of fibrinoly'sis in plasma. TAFI is a proenzyme, which is activated by proteoly tic cleavage to an active carboxypeptidase B-like enzyme (TAFIa, $35.8 \mathrm{kD}$ ) by thrombin/thrombomodulin and plasmin. Modulation of fibrinolysis occurs when TAFIa enzymatically removes C-teminal lysine residues of partially degraded fibrin, thereby inhibiting the stimulation of tissue plasminogen activator (t-PA) modulated plasminogen activation. TAFIa undergoes a rapid confomational change at $37^{\circ} \mathrm{C}$ to an inactive isofom called TAFIai. Potato tuber carboxy petidase inhibitor (PTCI) was shown to specifically bind to TAFIa as well as TAFIai. In this study, a novel immunoassay TAFIa/ai ELISA was used for quantitation of the two TAFI activation isoforms TAFIa and TAFIai. The ELISA utilizes PTCI as the capture agent and a double antibody sandwich technique for the detection. Low levels of TAFIa/ai antigen levels were detected in nomal plasma and elevated levels were found in hemophilia A plasnas. TAFIa/ai antigen represents a novel marker to monitor fibrinolysis and TAFIa/ai ELISA may be a valuable assay for studying the role of TAFI in normal hemostasis and in pathological conditions.
\end{abstract}

Key Words: TAFIa/ai. TAFI, TAFIa. TAFIai. Fibrinolysis

\section{Introduction}

Thrombin activatable fibrinolytic inhibitor (TAFI) [also known as plasma procarboxypeptidase $\mathrm{B}$ ( $\mathrm{PCPB}$ ). or $\mathrm{U}$ (PCPU)] is a $60 \mathrm{kD}$ glycoprotein present in human plasma that modulates fibrinoly sis in wo. TAFIa modulates fibrinoly sis by encymatically removing C-terminal lysine residues of partially degraded fibrin thereby inhibiting the stimulation of tissue plasminogen activator (t-PA) mediated plasminogen activation. ${ }^{1.6}$ TAFI is a proenzyme which is activated by proteoly tic cleavage at Arg-92 by thrombin to an $35.8 \mathrm{kD}$ carboxypeptidase B-like enzyme (TAFIa. Figure 1). Activation of TAFI by thrombin is accelerated over one thousand-fold by addition of thrombomodulin and $\mathrm{Ca}^{2-}$. TAFI provides a link between the coagulation and fibrinolytic pathways through reliance on the thrombin/thrombomodulin complex which activates TAFI as well as Protein C. Plasmin, the major protease that mediates fibrinolysis. also activates TAFI by cleavage at Arg-92. However, plasmin also cleaves TAFI at a second site in the C-terminal region at Arg-307 resulting in a tnuncated $44.3 \mathrm{kD}$ protein which lacks apparent biological activity."

TAFIa rapidly loses enzymatic activity through a temperature-dependent $\left(\mathrm{t}_{i}=10 \mathrm{~min}\right.$ at $\left.37^{\circ} \mathrm{C}\right)$ inactivation process. The inactivation of TAFIa is not due to a proteolytic process, but rather to a conformational alteration in the protein stnicture that can be followed by a change in the fluorescence spectnum of the protein. ${ }^{8}$ The confonmationally inactive form of TAFla is referred to as TAFlai $(35.8 \mathrm{kD})$. The temperaturedependent inactivation of TAFIa to TAFIai is believed to be the major mechanism by which TAFIa activity is modulated as no known natural inhibitors of TAFIa have been found in human plasma.

Polymorphisms in the TAFI gene have been shown to regulate the levels of TAFI protein in plasma. ${ }^{\text {g-11 }}$ TAFI plasma levels have been measured using functional and quantitative ELISA assays in nonnal ${ }^{12}$ populations and in various pathological conditions including liver disease. ${ }^{13}$ cancer. $^{14}$ DIC. ${ }^{15}$ arterial and venous thrombosis. ${ }^{16.18}$ The quantitation of TAFI in plasma has become problematic due to discordant results produced by the various assays used to measure TAFI polymorphisms. ${ }^{19-21}$ Recently, it has been recognized that the Thr325Ile poly morphism can alter the value of TAFI depending on the ELISA used. The differential reactivity of TAFI polymorphisms with some ELISAs causes in accurate reporting of the TAFI levels. Guimaraes et $a .^{22}$ showed that the Thr325Ile TAFI polymorphic proteins have the same reactivity using a conmercial chromogenic functional assay or an ELISA made using carefully selected pairs of monoclonal antibodies. The quantitation of TAFI by ELISAs can also be skewed by the differential reactivity towards different forms of TAFI (e.g. TAFI. the amino terminal activation peptide. TAFIa, and TAFIai) that may be present in the plasma. ${ }^{23}$

A clearer understanding of the role of TAFI in fibrinolytic processes can be gained by specifically measuring the individual TAFI proteins. The measurement of TAFI. the procarboxypeptidase. provides an estimate of the potential TAFI-dependent ant-fibrinolytic activity in plasma. Functional assays and ELISAs specific for TAFI have been utilized in several studies for quantitating the procarboxypeptidease in plasma(9, 11, 20, 21, 23, 24). Recently. TAFI was found in human seminal plasma, where TAFI might be involved with the coagulation and liquefaction of human semen for the protection of the seminal clot against lysis. ${ }^{25}$ Further insights into the biology of TAFI can be gained by measuring the TAFIa and TAFIai activation isofonms. The activation of TAFI is regulated by a number of factors including expression 
of genetic variants with different specific activities. gene polymorphisms that regulate circulating TAFI levels. thrombin and plasmin generation and thrombomodulin levels. Measuring TAFIa and/or TAFIai activation isoforms in plasma by immunoassay has been difficult because the protein sequences of the two activation isoforms are fully contained within the TAFI proenzyme protein sequence. Development of an immunoassay that specifically quantitates TAFIa and TAFIai isoforms in plasma would be a valuable tool for investigating the TAFI activation pathway

In the present studies. TAFIa/ai ELISA was used for the specific quantitation of TAFIa and TAFIai antigens in plasma. The assay is based upon the finding that conformationally inactive TAFIai isoform as well as TAFIa specifically bind to potato tuber carboxypeptidase inhibitor (PTCI). The TAFIa/ai ELISA uses PTCI-coated microtiter wells as the solid phase capture agent. Detection of the bound TAFIa or TAFIai is done using a two-step sandwich immunologic technique. Both TAFIa and TAFIai react equally well in the ELISA. whereas, TAFI (pro- enzyme) does not crossreact in the assay. Low levels of TAFIa/ai antigen was detected in normal plasma and signifiçantly higher levels were determined in plasmas from hemophilia A patients. The presence of TAFIa/ai antigen in plasma was confirmed by purification of the TAFIa/ai antigens using PTCI-Sepharose affinity column. The measurement of TAFIa/ai antigen by ELISA provides a novel plasma marker for monitoring the activation of TAFI and investigating the role TAFI plays in the regulation of fibrinolytic processes.

\section{Materials and Methods}

Materials. Citrated plasmas from hemophilia A and B were obtained from Diagnostic Support Services (West Barnstable. MA) and Biochemed Inc. (Atlanta. GA). Plasma was obtained after centrifugation at $2000 \mathrm{x}$ g for 15 minutes at $4^{\circ} \mathrm{C}$ and stored at $-80^{\circ} \mathrm{C}$ before usse. Pooled normal plasmas (PNP) were from Universal Reagents. Inc. (Indianapolis, IN). Acti- Chrome TAFI kits. PPACK thrombin and thrombomodulin were from American Diagnostica Inc. (Greenwich, CT). Purified human TAFI and anti-human TAFI monoclonal antibody was from Hematologic Technologies Inc. (Essex Junction. VT). TAFIdeficient plasma was from Affinity Biologics (Hamilton. Canada). PTCI was from Sigma Chemical Co. (St Louis. MO). Cyanogen bromide (CNBr)-activated Sepharose $4 \mathrm{~B}$ was from Pharmacia (New Brun- swick. NJ). Donkey anti-mouse IgGHRP was from Jackson Laboratories (Jackson, ME). Precast SDS-PAGE gels were from Invitrogen (Carlsbad. CA)

Preparation of TAFla and TAFlai. TAFI was activated by incubating alpha-thrombin (50 units/ml). thrombomodulin (3 units) $\mathrm{Ca}^{+}(5 \mathrm{mM})$, and TAFI $(50 \mu \mathrm{g})$ in $500 \mu \mathrm{l}$ of $100 \mathrm{mM}$ TRIS-HCl pH $7 .+$ for 20 minutes at room temperature. The reaction was stopped by addition of PPACK ( $1 \mu \mathrm{g})$ to stop the proteolysis of TAFI by thrombin. The generation of TAFIa carboxypeptidase B activity was confirmed using the ACTI CHROME TAFI activity assay. TAFIa was used immediately or stored frozen at $20^{\circ} \mathrm{C}$. TAFIai was prepared by incubating TAFIa for 2 hours at $37^{\circ} \mathrm{C}$ in a water bath. The TAFIai was determined to have lost $>98 \%$ carboxypeptidase B activity (data not shown) using the ACTICHROME TAFI assay.

Preparation of PTCI-Sephanose. A PTCI-Sepharose affinity colunu was prepared by mixing PTCI (25 mig) with activated $\mathrm{CNBr}-$ Sepharose ( $3 \mathrm{nl}$ of gel, Pharmacia. $\mathrm{NJ}$ ) in $3 \mathrm{ml}$ of $0.1 \mathrm{M} \mathrm{NaHCO}, 0.5 \mathrm{M} \mathrm{NaCl}_{2} \mathrm{pH} 8.3$ coupling buffer with gentle rotation over night. The PTCI-Sepharose was washed with coupling buffer and the unreacted $\mathrm{CNBr}$-sites were blocked by incubating with $0.1 \mathrm{M}$ Tris- $\mathrm{HCl}$ at $\mathrm{pH} 8.0$ for 2 hrs at room temperature. The PTCI-Sepharose column was sequentially washed with $0.1 \mathrm{M}$ acetate buffer. $0.5 \mathrm{M} \mathrm{NaCl}, \mathrm{pH}$ 4.0, and with $0.1 \mathrm{M}$ Tris- $\mathrm{HCl}, 0.5 \mathrm{M} \mathrm{NaCl}$. at $\mathrm{pH} 8.0$ and equilibrated with $20 \mathrm{mM}$ TRIS-HCl, pH 7.4 before use.

Affinity Chromatography with PTCI-Sepharose. TAFIa, prepared by treatment of TAFI with thrombin/thrombomodulin as described above, was applied onto the PTCI-Sepharose column. The colunn was washed with $20 \mathrm{mM}$ Tris. $\mathrm{pH} 7.4$ and the bound TAFIa protein was eluted with $0.5 \mathrm{M} \mathrm{NaCl}$ in $200 \mathrm{mM}$ Tris, $\mathrm{pH}-7.4$. Fractions $(1 \mathrm{ml})$ were collected and protein monitored at OD $280 \mathrm{~nm}$. TAFIa activity in the fractions was determined using ACTICHROME TAFI kit. The fractions were analyzed by SDS-PAGE and Western Blotting using a goat anti-human TAFI IgG-HRP polyclonal antibody for detection. TAFIai was affinity purified using the same procedures as for TAFIa. TAFIa/ai antigen was affinity purified from hemophilia A plasma. The plasma was diluted 50 -fold with $20 \mathrm{mM}$ Tris- $\mathrm{HCl}, \mathrm{pH} 7.4$ before application to the PTCISepharose affinity column. The same column washing and elution conditions were used as described above.

TAFLa ai ELISA. Dỵnax Immulon 4 HBX microtiter plates

Table 1. Quantitation of TAFIa/ai antigen in nomal plasma using TAFIa/ai ELISA

\begin{tabular}{lcc}
\hline \multicolumn{1}{c}{ SAMPLE } & $\mathrm{N}$ & MEAN \pm STD \\
\hline Pooled Nonmal Plasmá & 3 & $15.8 \pm 19.0 \mathrm{ng} / \mathrm{ml}$ \\
Normal Plasma & 16 & $18.6 \pm 17.1 \mathrm{ng} / \mathrm{ml}$ \\
\hline
\end{tabular}

Table 2. FVIII and TAFIa/ai levels in hemophilia plasmas.

\begin{tabular}{|c|c|c|c|c|}
\hline Sample & Age & Sex & $\begin{array}{l}\text { TAFIa/ai } \\
(\lfloor\mathrm{g} / \mathrm{ml})\end{array}$ & $\begin{array}{c}\text { FVIII } \\
(\% \text { activity })\end{array}$ \\
\hline 1 & 22 & $\$ .1$ & 30.6 & $<1$ \\
\hline 2 & 37 & $M$ & 39.0 & 23 \\
\hline 3 & 17 & $\mathrm{M}$ & 141.9 & $<1$ \\
\hline 4 & 31 & $\mathrm{~F}$ & 1730 & 18 \\
\hline 5 & 27 & $M$ & 81.7 & $<1$ \\
\hline 6 & 15 & $\$ .1$ & 80.7 & 5 \\
\hline 7 & 20 & $M$ & 125.3 & 17 \\
\hline 8 & 33 & $M$ & 138.2 & 11 \\
\hline 9 & 41 & M & $15+8$ & 35 \\
\hline 10 & 13 & $\mathrm{M}$ & $12: 2$ & 3 \\
\hline 11 & $\mathrm{NP}^{a}$ & NP & 78.6 & $<1$ \\
\hline 12 & $N P^{3}$ & NP & 95.3 & $<1$ \\
\hline 13 & $N P$ & NP & 65.9 & 9 \\
\hline 14 & NP & NP & 45.9 & 5 \\
\hline 15 & $\mathrm{NP}$ & NP & 18.9 & 16 \\
\hline 16 & NP & NP & 261 & $<1$ \\
\hline
\end{tabular}

not provided 


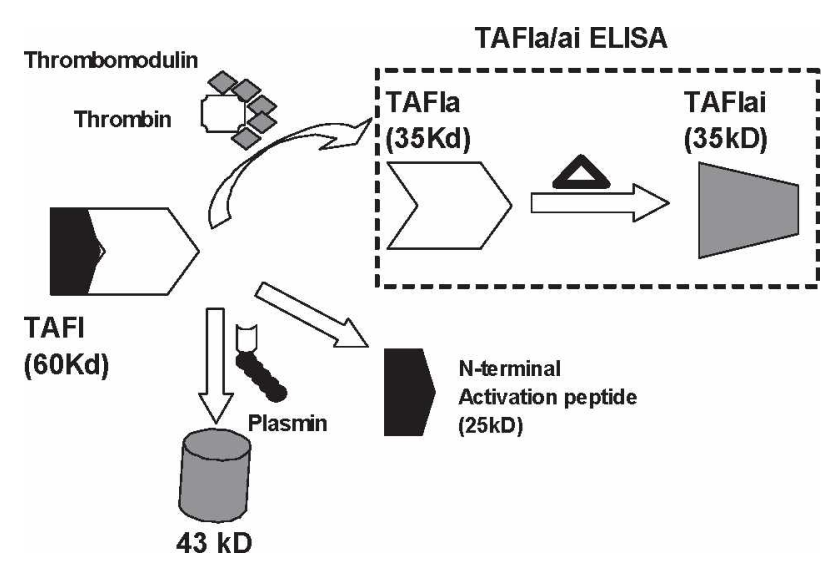

Figure 1. Major molecular tonns of TAFI in plasma. TAFI (zymogen, $60 \mathrm{kD}$ ) could be activated by cleavage at Arg-92 with thrombin thrombomodulin or plamin, yielding TAFIa (active form, $35 \mathrm{kD}$ ) and non-enzymatic for of $\mathrm{N}$-teminal activation peptide $(25 \mathrm{kD})$. Plasmin could cleave at alternative site, Arğ-307, yielding nuactive enzyme with intact $\mathrm{N}$-tenminal activation peptide $(43 \mathrm{kD})$. The open triangle indicated the inactivation factors, time and heat, of inactivating TAFIa to TAFIai. TAFIa/ai ELISA measured only TAFIa and TAFIai forns from altentative TAFI forms in plasma as indicated in the dotted box.

(Dynatech Lab. Inc.; Chantilly, VA) were coated with PTCI $(1 \mu \mathrm{g} / \mathrm{ml})$ and blocked with SuperBlock Blocking Buffer (Pierce Chemicals. IN). The standard curve was generated using two fold serial dilution of TAFIa in TAFI-deficient plasma or in phosphate buffered saline (PBS) containing $0.03 \%$ Tween 20. Human citrated plasma samples were diluted three-fold in buffer prior to being assayed. The TAFIa. TAFIai or diluted plasma samples $(100 \mu \mathrm{l})$ were added into the PTCI-coated microwells and incubated for 2 hours at room temperature. The wells were washed four times with phosphate buffered saline containing $0.03 \%$ Tween 20 . Antihuman TAFI monoclonal antibody $(0.5 \mu \mathrm{g} / \mathrm{ml})$ was added to each well and incubated for $1 \mathrm{~h}$ at room temperature. The microtiter wells were washed four times and $100 \mu \mathrm{l}$ donkey antimouse IgG-HRP of $(1: 5,000)$ was added to each well. After incubation for $l h$ the wells were washed and TMB substrate (Moss, MD) was added to each well. The color was developed for 10 minutes at room temperature and the reaction was stopped with $50 \mu \mathrm{l}$ of $0.5 \mathrm{M}$ sulfuric acid. The microtiter plate was read at $450 \mathrm{~nm}$ using a SpectroMax190 spectrophotometric plate reader (Molecular Devices Inc.. Sunn- yvale CA).

\section{Results}

Purification of TAFIa and TAFlai by PTCI-Sepharose Affinity Chromatography. After activation of TAFI with thrombin/thrombomodulin, the reaction mixture containing TAFIa was applied to a PTCI-sepharose affinity column. After washing with buffer. TAFIa bound to the column was eluted with Tris-HCl pH 7.4 buffer containing $0.5 \mathrm{M} \mathrm{NaCl}$. TAFIa carboxy peptidase activity was detected in fractions 2 to 5 (Figure 2A). SDS- PAGE analysis of fractions 2-5 showed a single protein band of molecular weight $35 \mathrm{kD}$ (Figure 2B). Unactivated TAFI did not bind to the PTCI- column and was identi-
A

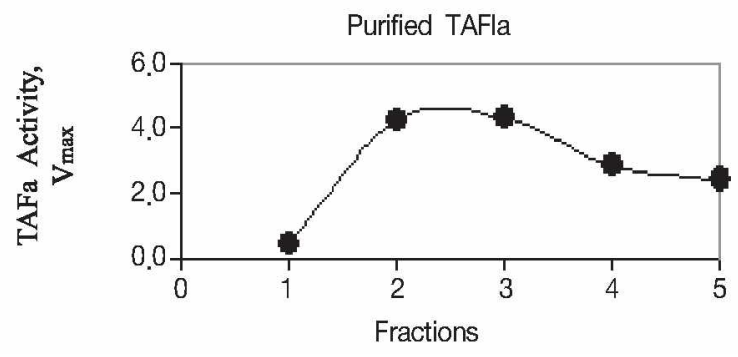

B

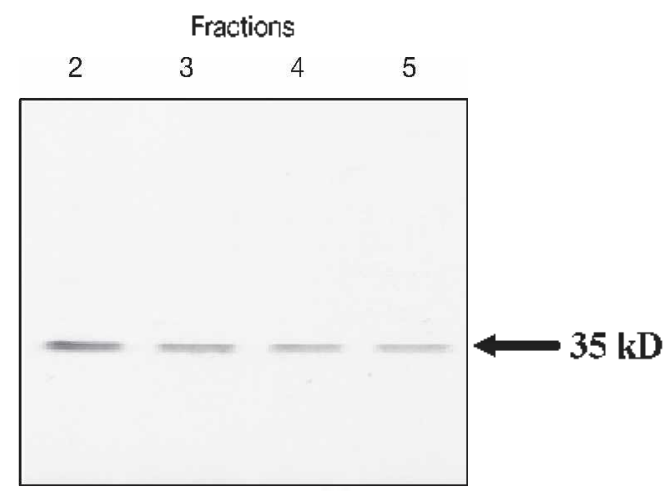

Figure 2. Aftunity purification of TAFIa/ai antigen. TAFI was activated for 20 minutes with thrombin/thrombomodulin and the mixture applied to PTCI-Sepharose aftinity column. The protein eluted in the $0.5 \mathrm{M}$ salt fractions was assayed for TAFI activity (panel A). The protein in tractions 2-5 was applied to SDS-PAGE gel and silverstained (Panel B).

fied by SDS-PAGE/Western blotting in the initial buffer wash (not shown). In a similar manner. TAFIai was prepared as described above and affinity purified on the PTCI-Sepharose column. The fractions from the $0.5 \mathrm{M} \mathrm{NaCl}$ wash did not contain carboxypeptidase B activity but a single $35 \mathrm{kD}$ TAFIai protein was present (data not shown). These results indicate TAFIai retains the ability to bind to PTCI although it has no carboxy peptidase activity.

Specificity of TAFlaai ELISA. An ELISA assay for the two TAFI activiation isoforms was used based on the ability of PTCI to bind strongly to both TAFIa and TAFIai. PTCIcoated microtiter plate was used as the solid-phase capture agent in the ELISA. Detection of TAFIa or TAFIai bound PTCI-coated microtiter well was accomplished using a dual antibody sandwich ELISA technique with an anti-human TAFIIgG monoclonal antibody as the primary detection antibody and a polyclonal donkey antimouse-IgG-HRP labelled second antibody. TAFIa were spiked into TAFI-deficient plasma at different concentrations and run in the ELISA (Figure 3). TAFIa was readily detected in the ELISA in a concentration dependent manner. The specificity of the ELISA was determined by spiking the TAFI-deficient plasma with increasing concentrations of TAFI. TAFI was not detected in the ELISA demonstrating the ELISA is specific for the activated form of TAFI. Although TAFIa and TAFIai have the same molecular weights. they have significantly different conformations. We determined the reactivity of the two isoforns towards the anti-TAFI antibody used in the ELISA (Figure 4). Both TAFIa and TAFIai are readily detected in TAFI-deficient 


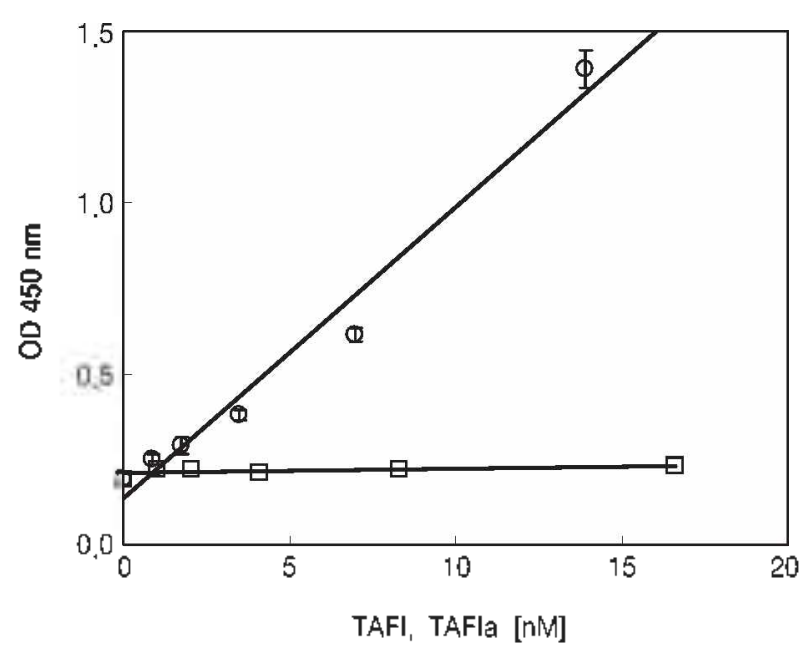

Figure 3. Specificity of TAFIa/ai ELISA. TAFIa (O) or TAFI (-) were spiked into TAFI-deficient plasma and the samples were run in the TAFIa/ai ELISA. Each point is the mean of duplicates. R-square values for TAFIa and TAFI were 0.9745 and 0.4393 , respectively.

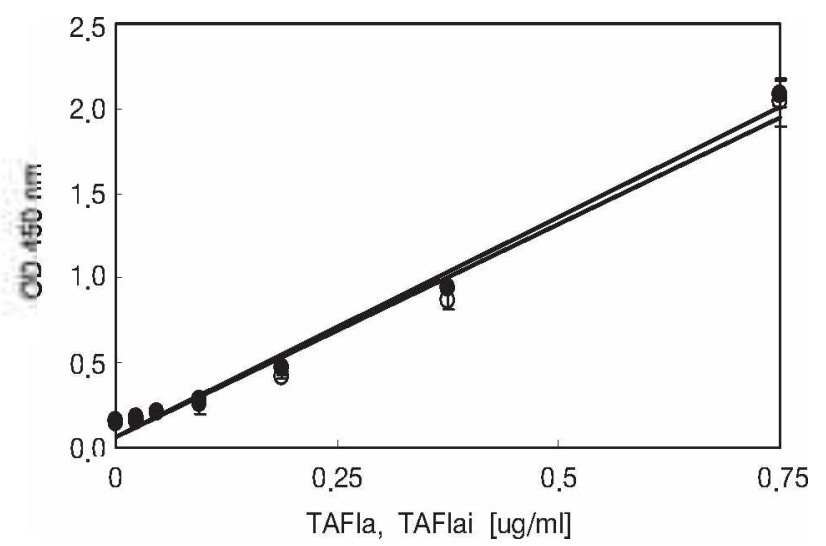

Figure 4. Reactivity of TAFIa and TAFIai proteins by ELISA. TAFIa (ם) or TAFIai ( ) protein was spiked into TAFI-deficient human plasma and nun in the ELISA assay. Each point is the mean of triplicates. R-square values for TAFIa and TAFIai were 0.9827 and 0.9882 , respectively.

plasma and the reactivity of TAFIai is equal to that of TAFIa in the ELISA. Since both isofonns forms are recognized equally well in plasma, the antigen recognized by the ELISA would be referred as "TAFIa/ai antigen"

Nomal plasma contains high levels (approximately 200 nM) of TAFI. To validate the ability of the ELISA to measure TAFIa/ai antigen in plasma in the presence of high concentrations of TAFI. we spiked nommal plasma with increasing amounts of TAFIa. As the amount of TAFIa spiked into normal plasma increased there was a corresponding increase in the amount of TAFIa measured in the ELISA (Figure 5). The sensitivity of the ELISA was approximately $1 \mathrm{nM}$ TAFIa in normal plasma. These findings show that the ELISA is specific for TAFIa/ai and is able to quantitate very low levels of TAFIa/ai antigen in the presence of high levels of TAFI normally found in plasma.

Measurement of TAFIa/ai Antigen in Nonmal Plasma by ELISA. Initial studies were perfonmed to determine if TAFIa/ai antigen was endogenously present in nomal plasma. We test-

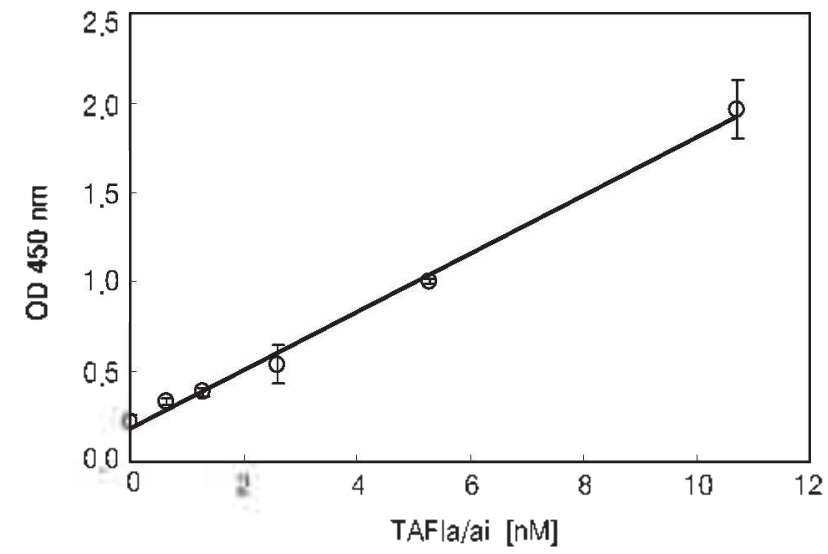

Figure 5. Measurement of TAFIa antigen spiked into nomal plasma. Pooled normal human plasma was spiked with increasing concentrations of TAFIa and run in the ELISA assay. Each point is the mearl of triplicates. R-square values was 0.9945.

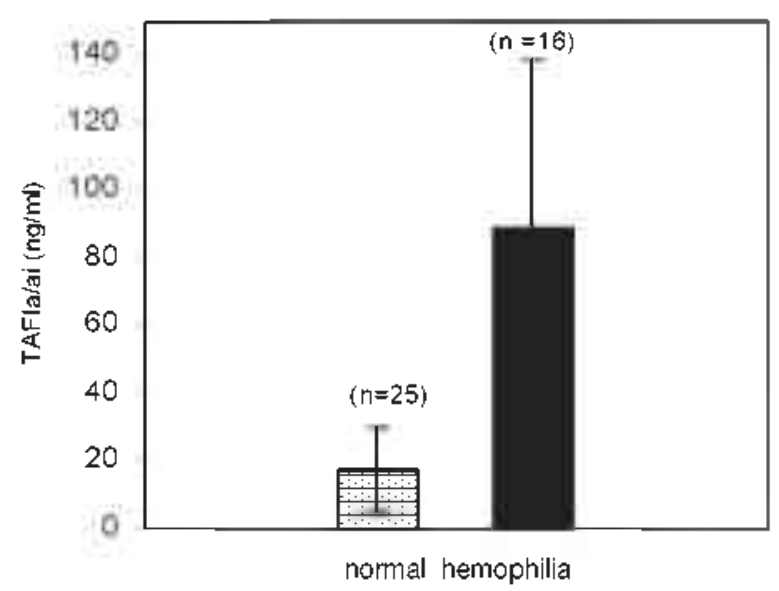

Figure 6. Quantitation of TAFIa/ai antigen levels in hemophilia A plasma. TAFTa/ai antigen levels were quantitated in twenty-six nomal plasmas (dotted har) and in sisteen plasmas from hemophilia A (black bar: using TAFIa/ai ELISA.

ed three commercial pooled normal plasmas (PNP) and sixteen conmercial citrated plasmas drawn from individuals for the presence of TAFIa/ai antigen. Low levels of TAFIa/ai antigen were determined in the three PNP samples tested (TABLE I). The TAFIa/ai antigen concentrations in individual nonmal plasmas ranged from 0 to $135 \mathrm{ng} / \mathrm{mll}$. The mean concentration of TAFIa/ai antigen was $18.6 \pm 17.1 \mathrm{ng} / \mathrm{mll}[0.5 \mathrm{nM}]$. Thus, $\mathrm{TAFIa} / \mathrm{ai}$ antigen is detectable at low levels in nomal plasma using the TAFIa/ai ELISA.

Elevated Levels of TAFLai in Hemophilia A. Increased fibrinolytic activity has been reported in hemophilia. ${ }^{26}$ It has also been observed that TAFI levels are depleted in hemophilia and may be related to up-regulation of TAFI activation." If TAFI activation was enhanced in hemophilia then one night expect to find increased levels of TAFIa and/or TAFIai in hemophilic plasma. We tested sixteen plasma samples from patients with hemophilia A for elevated levels of TAFIa/ai antigen using the ELISA (Figure 6). The mean TAFIa/ai antigen levels in hemophilia A plasmas was $88.9 \pm 49 \mathrm{ng} / \mathrm{ml}$. TAFIa/ai antigen was significantly elevated $(\mathrm{p}<0.05)$ as compared to 


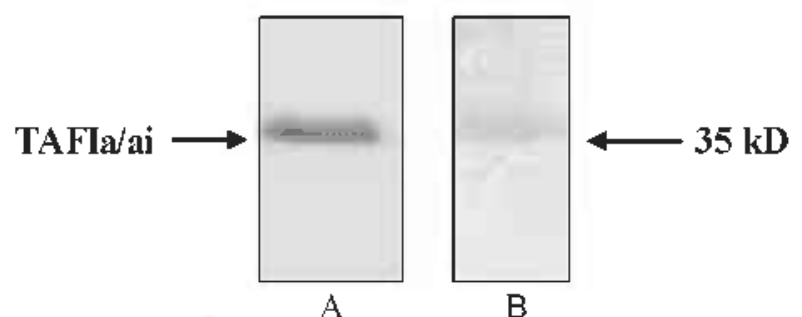

Figure 7. Identification of TAFIa/ai antigen in hemophilia plasma. TAFIa/ai was purified from hemophilia plasma by affinity chromatography on PTC'I-Sepharose as described in Methods section. Protein eluted in high salt was applied to SDS-PAGE gel and silverstaned for protein (lane A). Westem blot of the sanne protein was inmunostanned with anti-TAFI antibody (lane B).

levels in normal plasmas (see above). There was no apparent correlation between TAFIa/ai levels and FVIII activity levels in hemophilia samples (TABLE 11). The finding of almost 5-fold higher levels of TAFIa/ai antigen in hemophilia plasma suggests that TAFI activation pathway is up-regulated in hemophilia.

Punfication of TAFla/ai Antigen from Plasma. To confirm the presence of TAFIa/ai antigen in hemophilia plasma. we attempted to isolate the TAFIa/ai antigen from plasma using PTCI-affinity chromatograply. A hemophilia plasma sample that contained high levels of TAFIa/ai antigen by the ELISA was applied to the PTCI-Sepharose affinity column. After washing away unbound protein, the column was eluted with $0.5 \mathrm{M} \mathrm{NaCl}$ high salt buffer as described above. The protein eluted in the high salt buffer was run on SDS-PAGE and the Western blot was immunostained with a goat anti-TAFI antibody. A $35 \mathrm{kD}$ protein was immunostained using the TAFIspecific polyclonal antibody (Figure 7). These findings confirm the presence of the TAFIa/ai antigen $(35 \mathrm{kD})$ in human plasma by ELISA.

\section{Discussion}

Fibrinolysis is a highly complex hemostatic process involving multiple fibrinolytic enzymes and proteins. TAFI has been shown to be an important down-regulator of fibrinolysis through its ability to remove $\mathrm{C}$-terminal lysine groups from partially degraded fibrin. Fibrinolysis is most commonly monitored by measurement of fibrin degradation products and D-dimer. The role that TAFI plays in pathological hemostatic conditions has been based mainly upon measurement of the $60 \mathrm{kD}$ proenzyme TAFI. In order to modulate fibrinolysis. TAFI has to be activated by proteoly tic enzy'mes (e.g. thrombin, plasmin) to TAFIa, the active carboxy peptidase B enzyme. A greater understanding of the function of TAFI may be achieved by quantitation of the endproducts of the TAFI activation pathway-TAFIa and TAFIai. The purpose of this study was to use a new method for the specific quantitation of TAFIa and TAFIai protein in plasma.

It has been well documented that PTCI binds to TAFIa with ligh affinity and blocks carboxypeptidase $B$ activity. In this study enzymatically active TAFIa as well as its inactive isoform TAFIai was shown to bind to PTCI with high affinity (Figure 2). TAFIa undergoes significant conformational change leading to the enyzmatically inactive TAFIai isoform. However. the PTCI binding site of TAFIa remains intact as the protein undergoes conformational changes to the TAFIai isoform. Extensive protein structural analysis and crystallographic studies are required in order to fully elucidate how and where PTCI binds to TAFIa and TAFIai.

Two TAFI activation isofonns were measured based on the ability of PTCI to bind to TAFIa and TAFIai. The TAFIa/ai ELISA consists of a PTCI-coated microtiter well as the solidphase capture agent and a double antibody sandwich technique for detection of the bound TAFIa/ai antigen. A TAFIspecific monoclonal antibody was used as the primary detection antibody. The immunoassay was specifically recognized TAFIa and TAFIai and as there was no crossreactivity with the proenzyme TAFI (Figure 3). An excellent linear correlation was obtained with either TAFIa or TAFIai with a low: er limit of detection of approximately $1 \mathrm{nM}$. The TAFIa/ai ELISA measured TAFIa and TAFIai antigens with equal sensitivity (Figure 4). The presence of high levels of TAFI in normal plasma does not interfere with the measurement of TAFIa/ai antigen.

Meașurable levels of TAFIa/ai antigen were found in several pooled normal plasmas as well as in some of the individual normal plasmas using the TAFIa/ai ELISA (TABLE I). The mean amount of TAFIa/ai antigen in normal plasma was $18.6 \pm 17.1 \mathrm{ng} / \mathrm{ml}[0.5 \mathrm{nM}]$. Neill $e t$ a ${ }^{28}$ using a highly sensitive fluorescent activity assay and plasma collected under carefully controlled conditions found very low levels ( 10 $\mathrm{pM}$ ) of TAFIa to be constitutively present in normal plasma. The finding of TAFIa/ai antigen by ELISA in normal plasma would be consistent with TAFIa being constituitively produced during normal hemostasis. However. the level of TAFIa/ai antigen in plasma by ELISA is significantly higher than the amount of TAFla determined using a fluorescence activity assay. The difference in the two results may be due to the nature of the TAFI activation isoform measured by the different assays. TAFIa constitutively generated in wivo is likely to be rapidly converted to TAFlai at body temperature. If TAFIai has a longer half-life than TAFIa in plasma. then one would expect TAFIai to be found at higher levels in plasma. Therefore. the TAFIa/ai antigen measured in plasma by ELISA is likely to be comprised mainly of the inactive TAFIai isoform rather than the active TAFIa enzyme. We did not detect enzy matically active TAFIa in the plasmas using a sensitive in-house TAFIa fluorescence activity assay (data not shown) which further supports that TAFIai is measured by the ELISA.

The TAFIa/ai antigen is the endproduct of the TAFI activation pathway and therefore should be a very useful new marker for monitoring fibrinolysis in various pathological conditions. It has been suggested that fibrinolytic activity is enhanced in hemophilia and may contribute to the excessive bleeding in hemophilia ${ }^{26}$ Mosnier et $a l^{27}$ restored the down regulation of fibrinolysis in hemophilia plasma by addition of TAFI to the hemophiliac plasma. Reduction in TAFI plasma levels reported by Antovic et al ${ }^{29}$ provides further support that TAFI is involved in the regulation of hyperfibrinolysis in hemophilia A. Evidence of up-regulation of TAFI activation 
in the sixteen hemophilia A plasmas was found by measuring TAFIa/ai antigen level using the ELISA. TAFIa/ai antigen was almost 5 -fold higher (mean values $88.6 \pm 49 \mathrm{ng} / \mathrm{ml}$ ) in hemophilia A plasmas ( $p<0.05$ ) as compared to normal plasma (Figure 6). Isolation of the TAFIa/ai antigen from hemophiliac plasma by PTCI-affinity chromatography confinmed the presence of the TAFIa/ ai antigen detected in plasma by the ELISA (Figure 7). Similar findings of elevated TAFIa/ai antigen in hemophilia plasma were reported in a preliminary study that used a modified version of the current ELISA reported herein. ${ }^{31}$ The finding of increased levels of TAFIa/ai antigen supports the hypothesis that TAFI activation is up-regulated in hemophilia A. It can be hypothesized that the enhanced fibrinolytic activity in hemophilia is counteracted by up-regulation of the TAFI activation pathway which leads to the depletion of TAFI.

The generation of thrombin is very low in hemophilia A due to a genetic defect in Factor VIII. The up-regulation of TAFI activation in hemophilia would seem unusual when thrombin. the major activator of TAFI, is not efficiently generated. Hyperfibrinolysis is likely mediated through enhanced plasmin generation via t-PA mediated activation of plasminogen. Plasmin is also known to be an effective activator of TAFI. Thus. enlanced plasmin generation associated with hyperfibrinolysis in hemophilia may also result in enhanced activation of TAFI and the elevation levels of TAFIa/ai antigen in hemophilia. High levels of plasmin-antiplasmin complex found in hemophilia plasmas support this hypothesis (unpublished results). Further work is needed to more fully understand the role TAFI activation plays in hemophilia and fibrinoly sis.

In conclusion, a novel ELISA assay was used for measuring the two $35 \mathrm{kD}$ activation isoforms of TAFI in plasma. The assay is specific for TAFIa and TAFIai but not TAFI proenzyme. We found low levels of TAFIa/ai antigen in normal plasmas and significantly elevated levels of TAFIa/ai antigen in hemophilia A plasmas. The finding of high levels of TAFIa/ai antigen indicates an upregulation of TAFI activation pathway and an ongoing fibrinolytic process in hemophilia. TAFIa/ai antigen measured by TAFIa/ai ELISA represents a novel marker for monitoring fibrinolysisis. Quantitation of TAFIa/ai antigen levels in plasma should permit greater understanding of the role that TAFI plays in hemostasis and various pathological conditions such as hemophilia.

Acknowledgments. The authors acknowledge Dr. Richard Hart and Robert S. Greenfield for their supports.

\section{References}

1. Redlitz, A.; Tan, A. K.; Eaton, D. L.; Plow, E. F. J. Clin. Invest. $1995,96,2534$

2. Bajzar, L: Manuel, R:; Neisheim M E. J. Biol (Them. 1995, $270,1477$.
3. Baizar. L.; Morser. T.; Neisheim M E. J. Biol. Chem. 1996, 271, 166013.

4. Saldarov, D. V: Plow; E. F.: Rijken D. C. J. Biol Chem 1997, 272, 14477.

5. Wang, W.; Botta, M. B.: Bạzar, L.: Walker, T. B.: Neisheim, M. E. J. Biol. Chem. 1998, 273,27176.

6. Hendriks, D.: Wang, W:; Scharpe, S; Lommaert, M. P; van Sande. M. Biochim. Biopinys. Acta 1990, 1034, 86.

7. Mars, P. F; Dawson, P. E.; Bouma, B. N.; Meijers, J. C. Biochemistry 2002, 11,6688 .

8. Marı, P. F; Hackeng, T. M: Dawson, P. E; Grittin, T. H: Meijers, J. C.; Bouma, B. N. J. Biol. Chem 2000, 275, 12410.

9. Franco, R. F.; Fagundes, M. G.; Meijers, J. C.; Reitsma, P. H.; Lourento, D.; Morelli, V; Maftei, F. H.; Ferrari, L. C.; Piccinato, C. E.; Silva, W. A. Ir.; Zago, M. A. Hoenatologica. 2001, 86, 510.

10. Henry, M: Aubert, H.; Morange, P. E.; Narni, L.; Alessi, M. C. Tiret, L.; Tuhan-Vague, 1. Blood 2001, 97, 2053.

11. Kostka, H.; Kullisch, E.; Schellong, S.; Siegert, G. Clin Lab. 2003. 49,645 .

12. Stromqvist, M:; Schatteman, K.; Leurs, J;: Verkerk, R.; Andersson, I. O.; Tohansson, T; Scharpe, S.; Hendriks, D. Thromb Haemost. $2001,85,12$.

13. Van Thiel, D. H; George, M; Fareed, J. Thronb Hownost. 2001, 85, 667.

14. Meijers, T. C.; Oudijk, E. T. D.; Mosnier, L. O.; Bos, R.; Bouma, B. N.; Neutentuis, H K; Fijnlwer, R. Br: J. Howatol. 2000, 108, 518.

15. Watanabe, R; Wada, H; Watanabe, Y; Sakakura, M; Nakasaki, T.; Mori, Y.; Nishikawa, M.; Gabazza, E. C.; Nobori, T.; Shiku, H. Thromb Res. 2001, 104, 1 .

16. Silveira, A.; Schatteman, K.; Goossens, F; Moor, E.; Scharpe, S.; Stromqvist, M:; Hendriks, D.; Hamsten, A. Thromb Haenost. $2000,84,364$.

17. van Tilburg, N. H.; Rosendal, F. R.; Bertina, R. M. Blood 2000. 95,2855

18. Schatteman, K. A.; Goossens, F. T: Lelis, I.; Kasahara, Y; Scharpe, S. S.: Hendriks, D. F. Chn Chem. Lab 1 ted 2001, $39,806$.

19. Schneider, M.; Botta, M.: Stewart, R.: Rahman, M.; Koschinsky, M.: Nesheim, M. J. Biol. Chem. 2002, 277, 1021.

20. Gils, A.: Alessi, M. C.: Brouwers, E.: Peeters, M.: Mars, P: Leurs, T.: Bouma, B.; Hendriks, D.: Juhan-Vague, I.; Declerck, P. T. Arterioscler Thromb lasc Biol. 2003, 23, 1122.

21. Knoetler, R.; Ludwig, K.: Kostka, H.; Kuhlisch, E.; Siegert, G.; Suttorp, M. Semin Thromb Hemost. 2003, 29, 575 .

22. Guimaraes, A. H.: van Tilburg, N. H.: Vos, H. L.: Bertina, R. M.: Rijken, D. C. Br J. Haematol 2004, 124,659.

23. Tani, S; Akatsu, H.: Ishikawa, Y:; Okada, N.; Okada, H. Wicrobiol Immmol 2003, 47,295

24. Schatteman, K. A.: Goosens, F. J.: Scharpe, S. S.: Neels, H. M.; Hendriks, D. F. Clin. Chem. 1999, 45,807

25. L waleed, B. A.; Goyal, A.: Greentield, R. S.; Cooper, A. I. Blood Coagul. Hibrinolysis 2007, 18,449.

26. Grunewald, M.; Siegemund, A.; Grunewald, A.; Konegan, A.; Koksch, M.; Griesshammer, M. Haemophilia 2002, 8, 768.

27. Mosnier, L. O.: Lisman, T.; van den Berg, H. M.; Nielwenhuis, H. K.: Meijers, J. C.: Bouma, B. N. Thromb Haemost. 2001, 86, 1035.

28. Neill, E. K.; Stewart, R. J.: Schneider, M. M.; Nesheim, M. E. Anal. Biachent 2004, 330,332

29. Antovic, J.; Schulman, S.; Eelde, A.; Blomback, M. Hasmophilia $2001,7,557$.

30. Greentield, R. S.; Antovic J; An, J.; Blombeck, M.; An, S. S. A. Procedings of the $16^{\text {th }}$ Int 1 Congress Fibrinolysis and Prote olysis 2002; Abstr. $\mathrm{S} 49$ 